\title{
Correlation of Field Potentials with Redox-states of Cytochromes Recorded from the Olfactory Cortical Slice of Guinea Pig
}

\author{
Takanori FujI,* Ulrike HeInRICH,** Jürgen Hoffmann, ** \\ and Dietrich W. LÜBBERS** \\ *Department of Physiology, Kyoto Prefectural University of Medicine, \\ Kyoto, 602 Japan \\ **Max-Planck-Institut für Systemphysiologie, Rheinlanddamm 201, \\ D-4600 Dortmund 1, West Germany
}

\begin{abstract}
Summary Field potentials and the reflection spectra of cytochromes were continuously recorded from the olfactory cortical slice of a guinea pig which was incubated under the normoxic and anoxic conditions. The reduction of the oxidated spectra of the cytochromes occurred before the decrease of field potential heights and the oxidation of the reducted spectra occurred before their recovery. In a 3 min anoxic experiment, a $90 \%$ recovery of the cytochromes $a a_{3}, b$, and $c$, and the field potential amplitudes took place in this order after the restoration of oxygen.
\end{abstract}

Key Words: brain slice, redox-states of cytochromes, field potential.

A continuous monitoring of the normoxic and the anoxic states of mammalian brain slices was performed by recording electrical activities and by using a rapid scanning spectrophotometer (LüBBERS and NIESEL, 1957), which was non-destructive and favorable for examining the oxidated and reducted states of the cytochromes in mitochondria in living tissues. The correlation between the electrical activities of neuronal tissues and the functions of the cytochromes is of importance since the electrical activities were maintained by consuming adenosine triphosphate (ATP) produced by oxidative phosphorylation in mitochondria. So far as we know, there is scanty literature which dealt with the correlation between the reflection spectra of the cytochromes by means of the rapid scanning spectrophotometer and the electrical activities as the field potentials, recorded from the olfactory cortical slices of guinea pigs.

The experiments reported here were designed to examine the time courses of

Received for publication February 18, 1983

* To whom all correspondence should be addressed.

藤井崇知 
the redox-states of reflection spectra of the cytochromes and the field potentials, recorded from the brain slices incubated under the normoxic and the anoxic conditions. As for the field potentials from the olfactory cortical slices, many reports have been published (FUJII, 1977; FuJII et al., 1978).

Figure 1 shows the schematic drawing of an olfactory cortical slice of horizontal section and experimental arrangements. The brain slices (300 to $400 \mu \mathrm{m}$ thick) were prepared from the olfactory cortex of the guinea pigs from which the blood was previously replaced by the physiological saline solution, and the field potentials were measured under the "gas-blow and medium-flow" conditions (Fujil et al., 1970). Supramaximal stimulation was applied to the anterior part of the lateral olfactory tract (LOT) at a rate of $1 \mathrm{~Hz}$ throughout the experiment. A silver ball electrode was used for recording the field potentials from the surface of the olfactory tubercle. The field potentials consisted of an initial spike (IS) potential (the potential conducted through the LOT) and a negative (N) potential (extracellularly recorded population EPSP) (RichARDS and SERCOMBE, 1968). An indifferent silver wire electrode was set in contact with a nylon mesh on which the slice was placed. The temperature of the brain slices was kept at $37^{\circ} \mathrm{C}$. The composition of the incubation medium (Krebs-Ringer's solution) was (mM): $\mathrm{NaCl}$ (120); $\mathrm{KCl}(4.8) ; \mathrm{KH}_{2} \mathrm{PO}_{4}$ (1.2); $\mathrm{MgSO}_{4}$ (1.2); $\mathrm{CaCl}_{2}$ (2.6); $\mathrm{NaHCO}_{3}$ (26); Glucose (10); $\mathrm{pH}$ adjusted to 7.4 with bicarbonate buffer. The medium was equilibrated with a $95 \% \mathrm{O}_{2}+5 \% \mathrm{CO}_{2}$ gas mixture. When the brain slices were incubated under the anoxic condition, a $95 \% \mathrm{~N}_{2}+5 \% \mathrm{CO}_{2}$ gas mixture was used instead of the $95 \% \mathrm{O}_{2}+5 \% \mathrm{CO}_{2}$ gas mixture. The reflection spectra of cytochromes were measured by means of the rapid scanning spectrophotometer. In order to record a large amount of optical signal, it was necessary to place a strip of white polyethylene membrane $(0.13 \times 12 \times 7 \mathrm{~mm})$ beneath the brain slice and to place the tip of the light guide of the spectrometer on that portion of the brain slice whose opposite surface was in contact with the white membrane.

Figure 2 shows the time course of the redox-states of the reflection spectra of the cytochromes and those of the average values of the IS and the $\mathrm{N}$ potential heights obtained from eleven preparations of the guinea pigs. The initial values of the redox-states of the cytochromes $b, c$, and $a a_{3}$ were 38,74 , and $82 \%$, respectively. When the oxygen gas was replaced by the nitrogen gas, the oxidation degree in the brain slice began to decrease rapidly, until it reached the values ranging from $3 / 4$ to $2 / 3$ of the initial at $100 \mathrm{sec}$ after the replacement. It continued to decrease gradually and reached almost steady states at $3 \mathrm{~min}$ of the anoxic period. When the incubation gas was again replaced by oxygen, the redox-states started to increase rapidly, and it recovered $90 \%$ of the initial within 1 min after the oxygen gas supply was resumed.

On the other hand, the average values of the IS and the $\mathrm{N}$ potential heights decreased more slowly during the early period of anoxia than the redox-state of the reflection spectra did. As for the potentials, the $\mathrm{N}$ started to decline in am- 


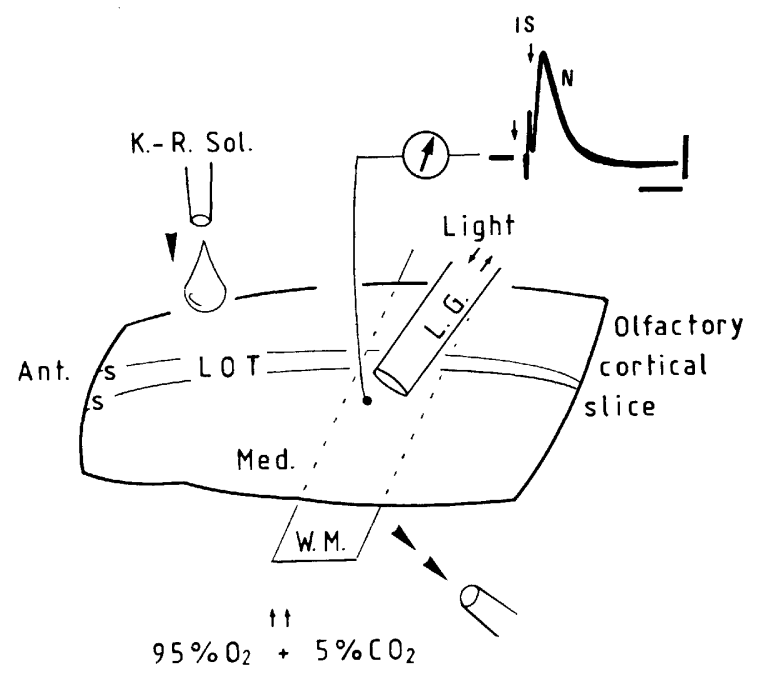

Fig. 1. Schematic drawing of olfactory cortical slice and experimental arrangements. The slice was superfused with Krebs-Ringer's solution (K.-R. sol.). S's indicate the location of the stimulation. Field potentials were recorded from the cortical surface of the olfactory tubercle, and the probe of light guide (L. G.) for measuring the spectrum of cytochromes was located near the recording electrode for the potentials. The arrow above the base line indicates stimulation. Time and voltage calibrations for the potentials are $10 \mathrm{msec}$ and $1 \mathrm{mV}$, respectively. Negativity upward. W. M., white polyethylene membrane; Ant., anterior; Med., medial.

plitude faster than the IS did at the early term of anoxia. The IS and the $\mathrm{N}$ potential heights decreased to $88 \pm 8 \%$ (mean \pm S. D.) and $83 \pm 6 \%$ at $80 \mathrm{sec}$ of the anoxic times, respectively, and then they still decreased to almost half of the control amplitude at $3 \mathrm{~min}$ of the anoxic time, i. e. $47 \pm 29 \%$ and $43 \pm 28 \%$, respectively. After the supply of the oxygen gas was resumed, both potentials began to increase in amplitude. The IS potential reached $90 \pm 17 \%$ of the control at $5 \mathrm{~min}$, and the $\mathrm{N}$ potential $90 \pm 9 \%$ at $7 \mathrm{~min}$.

With the start of the anoxic period, the reduction of the oxidated spectrum of the cytochromes occurred before the decrease of field potential heights, and with the commencement of re-normoxia, the oxidation of the reducted spectrum occurred before its recovery.

In order to compare the decreasing and the increasing rates of the cytochromes $b, c$, and $a a_{3}$ spectra at the beginning of anoxic and re-normoxic periods, respectively, the time courses of each oxidative spectrum were normalized. The decreasing rate of redox-state of cytochrome $a a_{3}$ was the slowest of the three, but its increasing rate was the fastest of the three at the commencement of renormoxia. The oxidated cytochrome $b$ spectrum varied inversely, compared with that of cytochrome $a a_{3}$. The cytochrome $c$ redox-state showed intermediate decreasing and increasing rates between those of cytochromes $a a_{3}$ and $b$. 


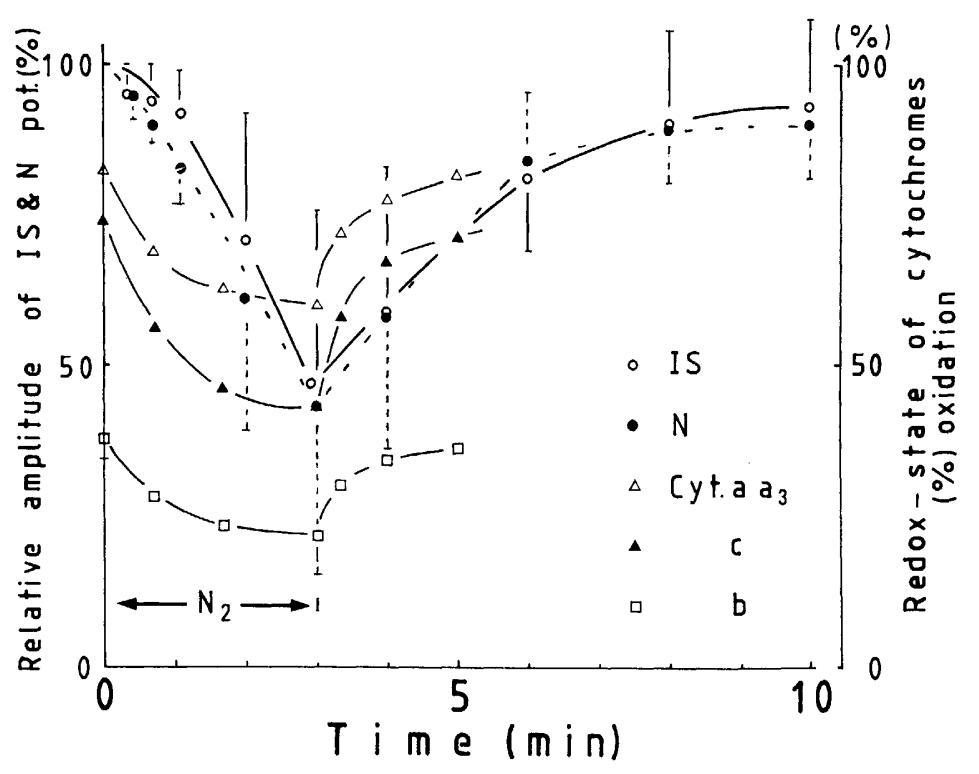

Fig. 2. Time courses of redox-states of the reflection spectra of cytochromes (Cyt.) and those of the IS and the $\mathrm{N}$ potentials. The brain slice was incubated under the condition of anoxia during the time indicated by $\mathrm{N}_{2}$. The percentage of oxidation of the individual cytochrome at the control shows different values. One hundred percent of the right vertical axis means $100 \%$ oxidation of cytochromes. Vertical solid and dotted lines represent the standard deviations of the IS and the $\mathrm{N}$ potential, respectively.

Under the normoxic experimental condition, the initial value of the cytochrome $a a_{3}$ was more oxidated than those of the other two. This finding supported the view that the cytochrome $a a_{3}$ located closest to oxygen of the three in the step of the respiratory chain (LEHNINGER, 1978).

The percentage of the redox-states of reflection spectra of the cytochromes decreased or recovered faster than that of the potential heights when the slices were incubated under the anoxic and re-normoxic conditions. These experimental results would indicate that the potential heights could be maintained during the early period of anoxia by using ATP stored before the commencement of anoxia and they could recover after the recovery of the energy production functions.

The $\mathrm{N}$ potential height decreased faster than the IS potential at the early period of anoxia. This result seemed to be reasonable because the IS was the compound action potential and the $\mathrm{N}$ was the transsynaptic one. Many processes for generating the latter, such as the synthesis, the mobilization and the release, of the transmitter substances, are sensitive to the decrease of ATP amount in the brain slices.

The reflection spectra represented the redox-state of cytochromes in the mitochondria of the whole layers of the brain slice. Although the electrical activity of the IS and the N potential is supported by ATP produced by the biochemi- 
cal events in the nervous tissues, we do not have sufficient data as yet to explain the mechanism of the time course of electrical activity by the time course of chemical events under the sequence of anoxic and re-normoxic conditions. Further investigation for elucidating the correlation between the electrical activity and the biochemical events in the nervous tissues would be needed.

\section{REFERENCES}

FUנII, T. (1977) Effects of cooling on guinea pig olfactory cortex maintained in vitro. Electroencepharogr. Clin. Neurophysiol., 43: 238-247.

FujI, T., Kubo, S., and IwaSE, Y. (1970) Gas-blow and medium-flow method for stable recording of electrical activities in the sliced mammalian cerebral tissue in vitro. J. Physiol. Soc. Jpn., 32: 333-334.

FujI, T., Murayama, K., and IbaTA, Y. (1978) The postnatal development of cortical structures and electrical activities in the guinea pig olfactory cortex slice. Brain Res., 142: 546-550.

LEHNINGER, A. L. (1978) Oxidation-reduction enzymes and electron transport. In: Biochemistry, 4th ed., Worth Publishers, Inc., Chap. 18.

LÜBBERS, D. W. and NIESEL, W. (1957) Ein Kurzzeit-Spektralanalysator zur Registrierung rasch verlaufender Änderungen der Absorption. Naturwissenschaften, 44: 60.

Richards, D. C. and Sercombe, R. (1968) Electrical activity observed in guinea-pig olfactory cortex maintained in vitro. J. Physiol. (Lond.), 197: 667-683. 